\title{
Lapatinib-resistant cancer cells possessing epithelial cancer stem cell properties develop sensitivity during sphere formation by activation of the ErbB/AKT/cyclin D2 pathway
}

\author{
YUICHI OHNISHI $^{1,2}$, HIROKI YASUI ${ }^{1,2}$, KENJI KAKUDO ${ }^{2}$ and MASAMI NOZAKI ${ }^{1}$ \\ ${ }^{1}$ Department of Cell Biology, Research Institute for Microbial Diseases, Osaka University, Suita, Osaka 565-0871; \\ ${ }^{2}$ Second Department of Oral and Maxillofacial Surgery, Osaka Dental University, Hirakata, Osaka 573-1121, Japan
}

Received April 26, 2016; Accepted August 26, 2016

DOI: 10.3892/or.2016.5073

\begin{abstract}
Lapatinib, a dual inhibitor of epidermal growth factor receptor (EGFR)/ErbB2, has antiproliferative effects and is used to treat patients with ErbB2-positive metastatic breast cancer. In the present study, we examined the effects of lapatinib on growth of oral and prostate cancer cells. Oral squamous cell carcinoma (OSCC) cell lines HSC3, HSC4 and $\mathrm{Ca} 9-22$ were sensitive to the antiproliferative effects of lapatinib in anchorage-dependent culture, but the OSCC cell lines KB and SAS and the prostate cancer cell line DU145 were resistant to lapatinib. Phosphorylation levels of EGFR in all cell lines decreased during lapatinib treatment in anchorage-dependent culture. Furthermore, the phosphorylation levels of ErbB2, ErbB3 and Akt and the protein levels of cyclin D1 were decreased by lapatinib treatment of HSC3, HSC4 and Ca9-22 cells. ErbB3 was not expressed and cyclin D1 protein levels were not altered by lapatinib treatment in KB, DU145 and SAS cells. The phosphorylation of ErbB2 and AKT was not affected by lapatinib in SAS cells and was not detected in KB and DU145 cells. Lapatinib-resistant cell lines exhibited sphere-forming ability, and SAS cells developed sensitivity to lapatinib during sphere formation. The phosphorylation levels of ErbB2 and AKT and protein levels of cyclin D2 increased during sphere formation of SAS cells and decreased with lapatinib treatment. In addition, sphere
\end{abstract}

Correspondence to: Dr Masami Nozaki, Department of Cell Biology, Research Institute for Microbial Diseases, Osaka University, 3-1 Yamadaoka, Suita, Osaka 565-0871, Japan

E-mail: mnozaki@biken.osaka-u.ac.jp

Abbreviations: CSCs, cancer stem cells; EGFR, epidermal growth factor receptor; EMT, epithelial-to-mesenchymal transition; FBS, foetal bovine serum; OSCC, oral squamous cell carcinoma; TBS-T, TBS/Tween-20; TKI, tyrosine kinase inhibitor

Key words: AKT, cancer stem cells, cyclin D1, cyclin D2, EGFR, ErbB, lapatinib, sphere formation of SAS cells was inhibited by the AKT inhibitor MK2206. AKT phosphorylation and cyclin D2 levels in SAS spheres were decreased by MK2206 treatment. SAS cells expressed E-cadherin, but not vimentin and KB cells expressed vimentin, but not E-cadherin. DU145 cells expressed vimentin and E-cadherin. These results suggested that phosphorylation of EGFR and ErbB2 by cell detachment from the substratum induces the AKT pathway/cyclin D2-dependent sphere growth in SAS epithelial cancer stem-like cells, thereby rendering SAS spheres sensitive to lapatinib treatment.

\section{Introduction}

The epidermal growth factor receptor (EGFR) family members, particularly EGFR and ErbB2, are targets of anticancer reagents, since their deregulated expression and activation play important roles in the initiation and progression of human cancer (1-4). Many types of tyrosine kinase inhibitors (TKIs) targeting EGFR have been clinically used since 2002 (5-8). Among them, the EGFR/ErbB2 dual inhibitor lapatinib is used to treat ErbB2-positive breast cancer. Despite intensive efforts investigating a large number of ligands identified for EGFR, ErbB3 and ErbB4, no direct ligand for ErbB2 binding has been identified. However, ErbB2 dimerizes with other ErbB receptors and acts as a co-receptor (9), and overexpression of ErbB2 can induce transformation of cells without the ligand (10). In addition, since heterodimeric formation of ErbB2 with other ErbBs can enhance ligand binding, receptor tyrosine phosphorylation, and cell proliferation compared with EGFR homodimers, lapatinib has better efficacy than those of single inhibitors of EGFR signal transduction for preventing tumour growth and survival (11). However, the mechanisms and efficacy of lapatinib have not been elucidated in systems other than breast cancer.

The clinical response of lapatinib is only $24 \%(12,13)$. Not all ErbB2 positive breast cancer cells respond to lapatinib treatment, and resistance to lapatinib develops in some patients and/or tumours during chronic exposure to the drug $(14,15)$. Since the therapeutic efficacy of lapatinib is affected by intrinsic and acquired resistance, it is important to identify the mechanism(s) conferring resistance to the drug. Several 
studies have previously reported the possible mechanisms of resistance to lapatinib. Since increased expression of the membrane receptor tyrosine kinase AXL conferred resistance to lapatinib, combination with the AXL inhibitor could particularly reverse resistance to lapatinib (14). In addition, Ras induced resistance to lapatinib and could overcome this resistance by MEK inhibition (16). Markedly, MCL-1, a member of the Bcl-2 family, induced lapatinib resistance and inhibition of MCL-1 restored the sensitivity of lapatinib toxicity via BAK-dependent autophagy $(17,18)$. It has also been reported that epithelial-to-mesenchymal transition (EMT) plays important roles in lapatinib resistance in ErbB2-overexpressing breast cancer cell lines (19). Since other mechanisms that induce lapatinib resistance are not well characterized, further investigations are needed.

We previously reported that EGFR signalling is not involved in anchorage-dependent growth of the cancer stem-like cell line, SAS, but contributes to anchorage-independent growth. Thus, SAS monolayer cultures are resistant to the EGFR-specific antibody cetuximab and to the EGFR TKI AG1478, but aggregated SAS cultures become sensitive to these reagents (20). Therefore, in the present study, we investigated the effects of the EGFR/ErbB2 dual inhibitor, lapatinib, on monolayer and sphere cultures of cancer stem-like cells.

\section{Materials and methods}

Cell culture and reagents. OSCC cell lines, HSC3, HSC4, SAS, KB and Ca9-22, and the prostate cancer cell line DU145 were purchased from RIKEN BioResource Center (Ibaraki, Japan). Cells were cultured in Dulbecco's modified Eagle's medium (DMEM) supplemented with $10 \%$ (v/v) foetal bovine serum (FBS) at $37^{\circ} \mathrm{C}$ in a humidified atmosphere of $5 \% \mathrm{CO}_{2}$. DMEM and FBS were purchased from Gibco (Life Technologies, Tokyo, Japan). Antibodies used included anti-EGFR, anti-phospho-Tyr1068-EGFR, anti-ErbB2, anti-phospho-Tyr1221/1222-ErbB2, anti-ErbB3, anti-phosphoTyr1289-ErbB3, anti-AKT, anti-phospho-S473-AKT (all from Cell Signaling Technology, Tokyo, Japan), antiERK, anti-phospho-Tyr204-ERK (both from Santa Cruz Biotechnology, Santa Cruz, CA, USA), anti-cyclin D1 (Abcam, Tokyo, Japan), anti-cyclin D2 (Cell Signaling Technology), anti-E-cadherin, anti-N-cadherin (both from Takara, Shiga, Japan), anti-vimentin (Merk Millipore, Damstadt, Germany), and anti- $\alpha$-tubulin (Sigma-Aldrich, Tokyo, Japan). Lapatinib (GW572016) and MK2206 were purchased from Selleckchem (Houston, TX, USA).

Cell proliferation assay. Human cancer cells (1x10 $3 /$ well) were seeded into 96 -well plates. After $24 \mathrm{~h}$ of growth, lapatinib was added at the indicated concentrations, and growth continued for an additional 1, 4 or 6 days. Cell proliferation was assessed using the CellTiter $96{ }^{\circledR}$ Non-Radioactive Cell Proliferation assay (Promega, Tokyo, Japan). All experiments were performed in triplicate.

Sphere formation cultures. Cells $\left(1 \times 10^{3}\right)$ were seeded into each well of ultra-low attachment 6-well plates (Corning, NY, USA) and cultured in DMEM supplemented with $10 \%$ (v/v) FBS at $37^{\circ} \mathrm{C}$ under $5 \% \mathrm{CO}_{2}$.
Western blotting. Cells were washed with phosphatebuffered saline (PBS), and then lysed with RIPA buffer consisting of $150 \mathrm{mM} \mathrm{NaCl}, 10 \mathrm{mM}$ Tris- $\mathrm{HCl}, \mathrm{pH} 8.0,1 \%$ (v/v) Nonidet P-40, $0.5 \%$ (w/v) deoxycholic acid, 0.1\% (w/v) SDS, and $5 \mathrm{mM}$ EDTA with $1 \mathrm{X}$ Halt ${ }^{\mathrm{TM}}$ Protease Inhibitor Cocktail and $1 \mathrm{X}$ Halt ${ }^{\mathrm{TM}}$ Protein Phosphatase Inhibitor (both from Thermo Fisher Scientific, Yokohama, Japan). The protein concentration of the lysates was determined using the $\mathrm{BCA}^{\mathrm{TM}}$ Protein Assay kit (Thermo Fischer Scientific), and equal amounts of protein were subjected to SDS-polyacrylamide gel electrophoresis. The separated proteins were transferred electrophoretically onto ClearTrans ${ }^{\circledR}$ polyvinylidene fluoride (PVDF) membranes (Wako Chemical, Tokyo, Japan). Non-specific binding was blocked by incubation in $5 \%(\mathrm{w} / \mathrm{v})$ bovine serum albumin in TBS/Tween-20 (TBS-T) for $1 \mathrm{~h}$ at room temperature. Membranes were probed with antibodies in TBS-T overnight at $4^{\circ} \mathrm{C}$, and then incubated with horseradish peroxidase-conjugated secondary antibody. Antibody-antigen complexes were detected by the ECL Plus Western Blotting Detection Reagent (GE Healthcare, Little Chalfont, UK).

\section{Results}

Lapatinib reduces proliferation of oral cancer cell lines lacking sphere formation capability. To assess the effect of lapatinib on cancer cells, we treated oral squamous cell carcinoma (OSCC) cell lines, HSC3, HSC4, SAS, KB and Ca9-22 and the prostate cancer cell line DU145 with lapatinib at various concentrations and determined growth at each culture day using the 3-(4,5-dimethylthiazol-2-yl)-2,5-diphenyltetrazolium bromide assay, as shown in Fig. 1A. Lapatinib at $1 \mu \mathrm{M}$ inhibited the proliferation of HSC3, HSC4 and Ca9-22 cells after 4 days of culture, but even $5 \mu \mathrm{M}$ lapatinib did not inhibit the proliferation of KB, SAS and DU145 cells during 6 days culture (Fig. 1A). Thus, HSC3, HSC4 and Ca9-22 cells were lapatinib-sensitive, and KB, SAS and DU145 cells were lapatinib-resistant.

Previous studies reported that lapatinib reduced the formation of mammospheres and proliferation of the progenitor/stem-enriched ductal carcinoma in situ population regardless of the ErbB2 status (21). To determine the correlation of lapatinib resistance with cancer stemness, we examined the sphere formation capacities of these cell lines. A suspension of $1 \times 10^{3}$ cells was cultured in ultra-low attachment 6 -well plates for 6 days. HSC3, HSC4 and Ca9-22 cells remained single cells in floating cultures, while KB, DU145 and SAS cells formed spheres (Fig. 1B). These results suggested that lapatinib-sensitive cells lack stem cell properties, while lapatinib-resistant cells exhibit cancer stem cell-like characteristics.

Lapatinib inhibits phosphorylation of ErbBs and AKT, and protein expression of cyclin D1 in sensitive cells. To investigate the effect of lapatinib on these cell lines at the molecular level, we extracted the protein from cells treated or not treated with lapatinib and western blotting was performed. Lapatinib reduced the phosphorylation level of EGFR in all cell lines. Phosphorylation of ErbB2 was detected in HSC3, HSC4, SAS and Ca9-22 cells and was reduced by lapatinib treatment. Phosphorylation of ErbB3 was detected in HSC3, HSC4 and Ca9-22 cells and reduced by lapatinib treatment (Fig. 2). 

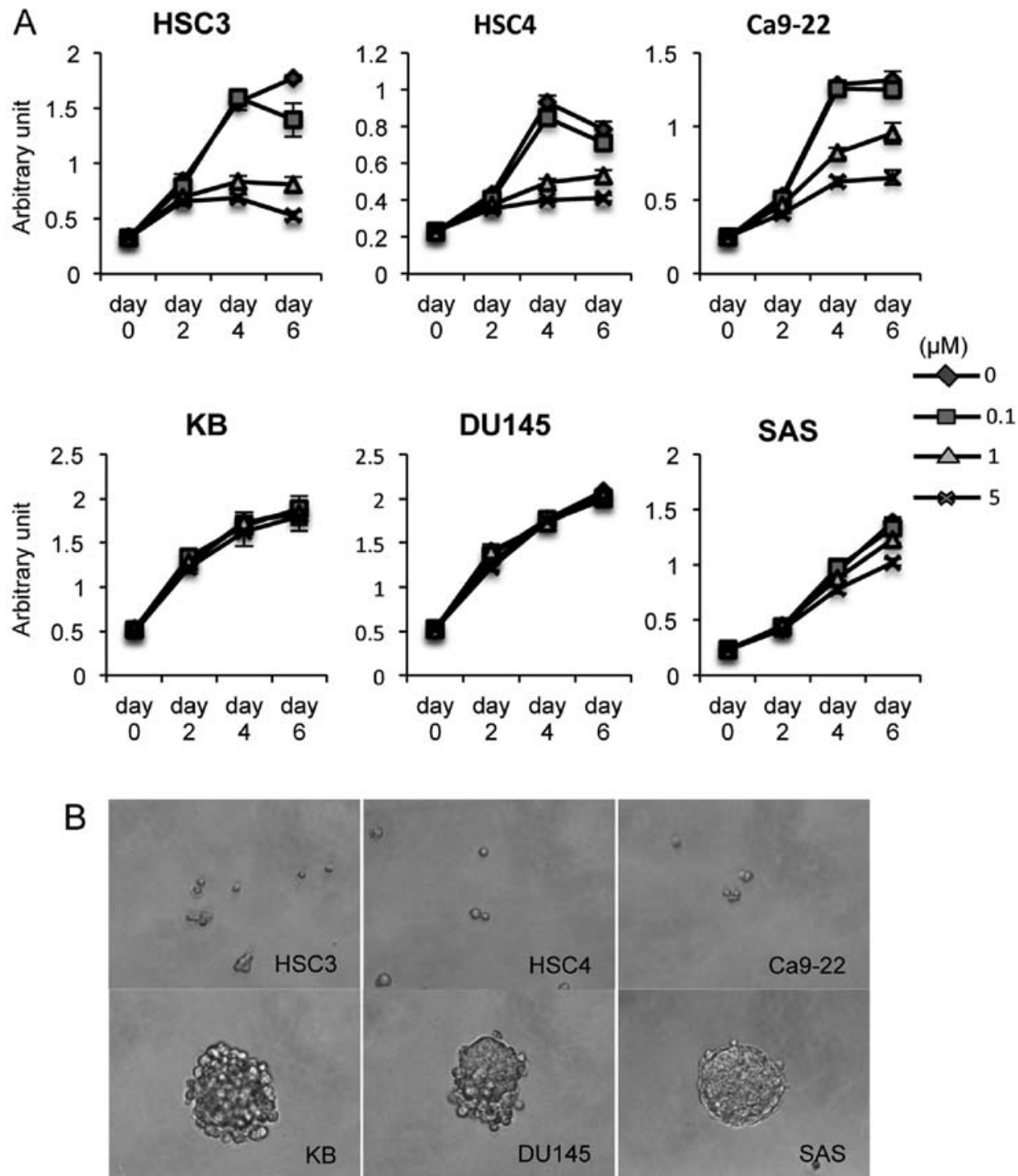

Figure 1. Lapatinib reduces the growth of cancer cells lacking sphere formation capability. (A) Each cell line was cultured in lapatinib at the indicated concentrations. The [3-(4,5-dimethylthiazol-2-yl)-5-(3-carboxymethoxyphenyl)-2-(4-sulfophenyl)-2H-tetrazolium] assay was performed on each day of culture. (B) Phase-contrast photomicrographs of cell masses derived from single cells; 1x10 3 HSC3, HSC4, Ca9-22, KB, DU145 and SAS cells were grown on ultra-low attachment 6-well plates.

These results indicated that growth inhibition by lapatinib correlated with inhibition of ErbB phosphorylation, including phosphorylation of EGFR, ErbB2 and ErbB3, in HSC3, HSC4 and $\mathrm{Ca} 9-22$ cells.

We next examined the phosphorylation of AKT and ERK, which are downstream effectors of the ErbB family signalling pathway. AKT phosphorylation was reduced by lapatinib treatment in HSC3, HSC4, and Ca9-22 cells, but not in SAS cells. Changes in AKT phosphorylation levels were not detected in KB or DU145 cells, since the phosphorylation levels were extremely low in untreated cells. The ERK phosphorylation level was decreased in HSC4 cells, increased in HSC 3 cells, and not altered in other cells by lapatinib treatment (Fig. 2).

The cell cycle involves a series of tightly controlled events that drive DNA replication and cell division during cell proliferation (22). The progression from G1 to $S$ is a critical checkpoint in protecting the cell from abnormal replication, and a key regulator of this process is cyclin D (23). A variety of mitogenic signalling pathways upregulate the expression of these cyclins. Thus, we examined cyclin D1 and D2 levels in lapatinib-treated cells. The cyclin D1 level was reduced by lapatinib treatment in $\mathrm{HSC} 3$, HSC4, and Ca9-22 cells. The cyclin D2 level was decreased in HSC3 cells, with no change noted in HSC4 or SAS cells. Cyclin D2 protein was not detected in KB, Ca9-22 and DU145 cells (Fig. 2). These results suggested that lapatinib inhibits phosphorylation of ErbBs, leading to suppression of the AKT cyclin D1 pathway, thereby reducing growth of $\mathrm{HSC} 3, \mathrm{HSC} 4$ and $\mathrm{Ca} 9-22$ cells.

Sphere formation of SAS cells via activation of the ErbB/AKT/ cyclin D2 signalling pathway is inhibited by lapatinib. We previously reported that anchorage-dependent growth of SAS cells is resistant to EGFR inhibitors, cetuximab and AG1478, but anchorage-independent growth becomes sensitive to these inhibitors (20). These results prompted us to examine the effects of lapatinib on sphere formation in KB, SAS and DU145 cancer cell lines possessing sphere formation capabilities. We first investigated changes in protein levels during 


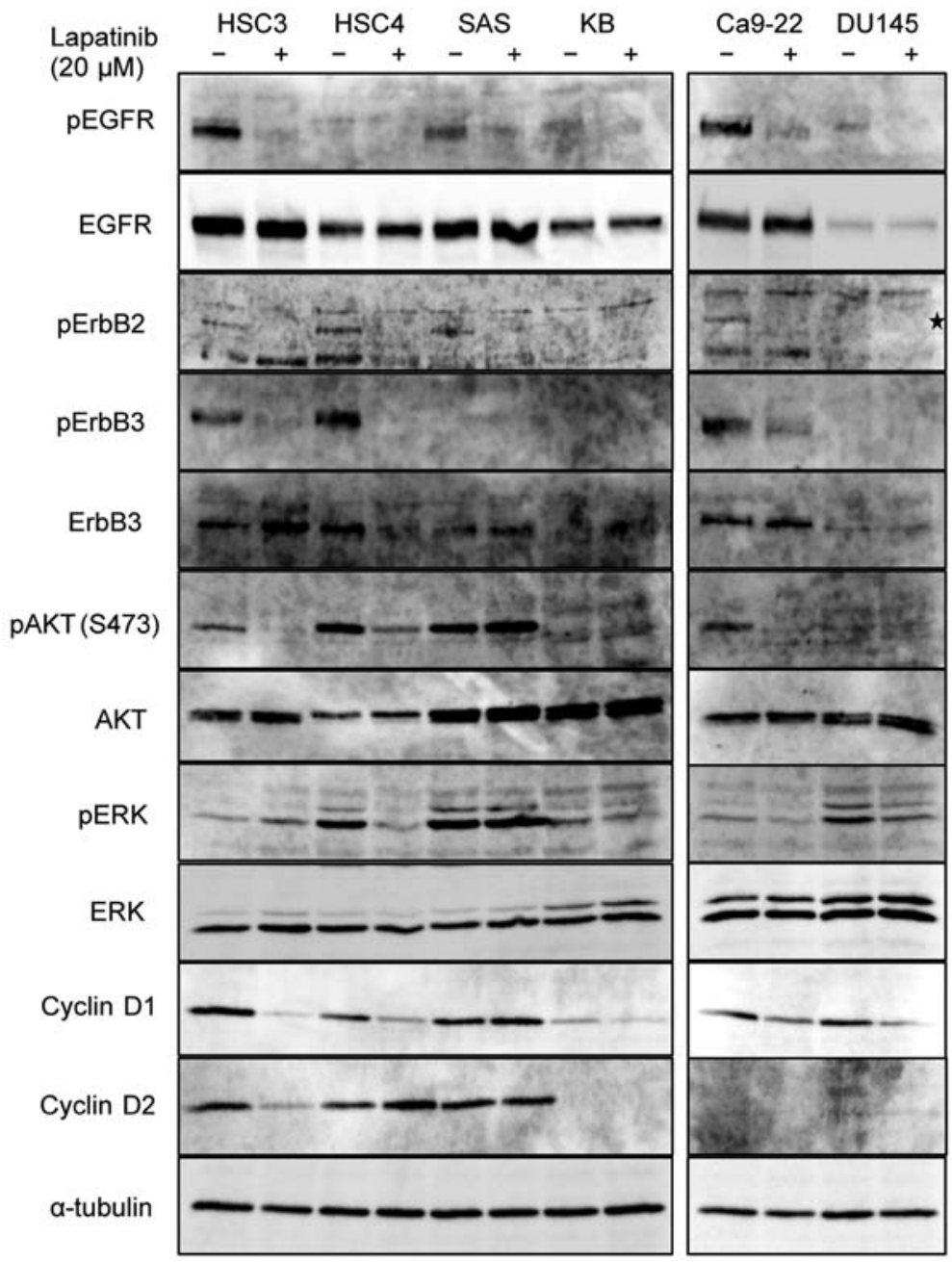

Figure 2. The effects of lapatinib on the activation states of the ErbB family and downstream AKT and ERK and on cyclin D2 protein levels were determined by western blotting. $\alpha$-tubulin was used as a loading control. Asterisk indicates the sieze of pErbB2 bands.

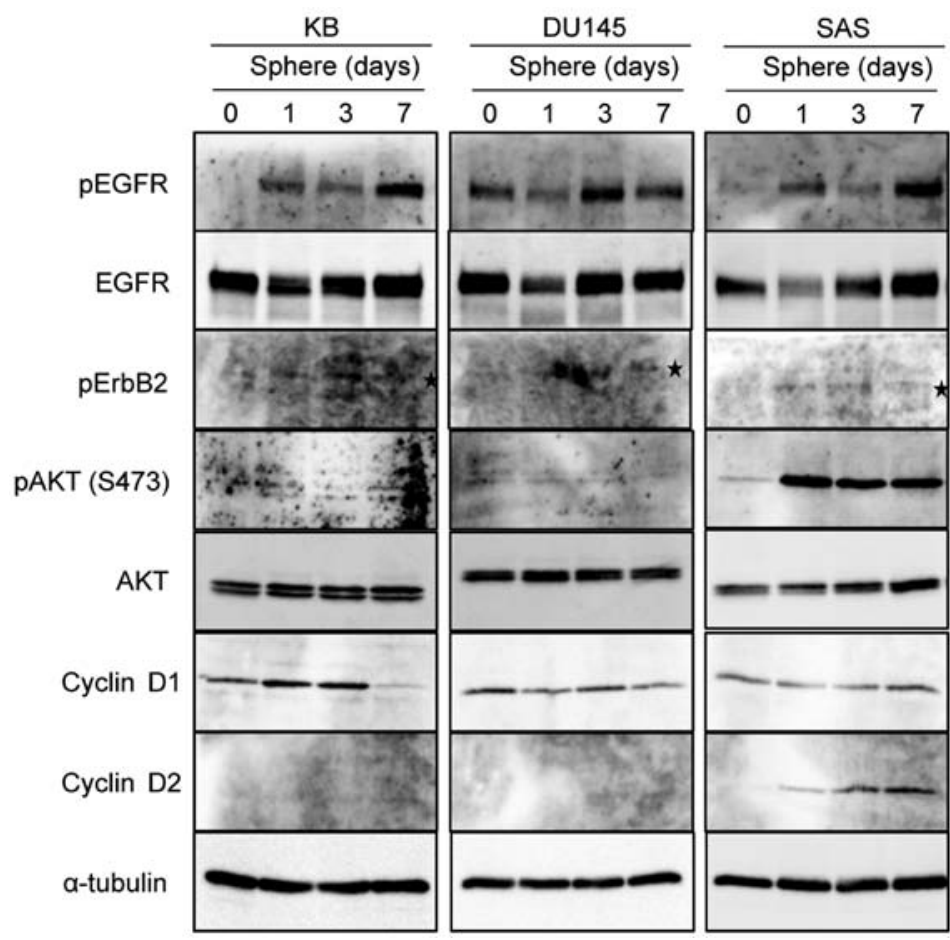

Figure 3. Spheres on each culture day were generated from KB, DU145 or SAS cells and the levels of pEGFR, EGFR, pErbB2, pAKT, AKT, cyclin D1 and D2 were determined by western blotting. Asterisks indicate the sieze of pErbB2 bands. 
A KB

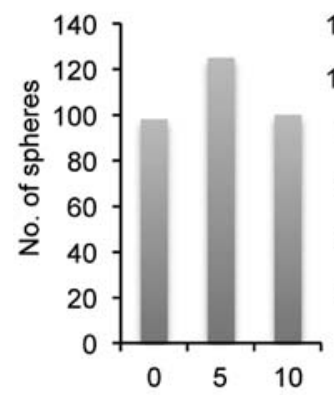
DU145

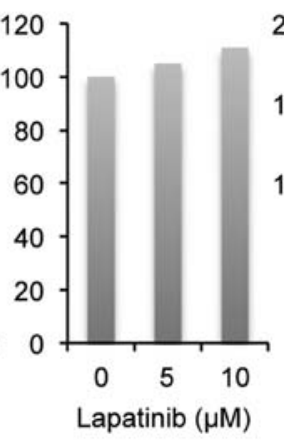

SAS

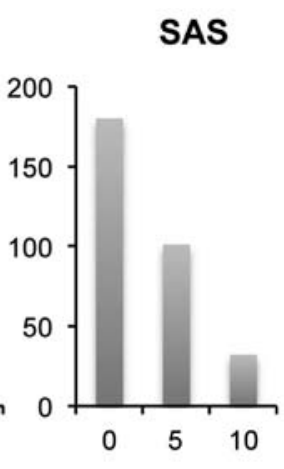

B

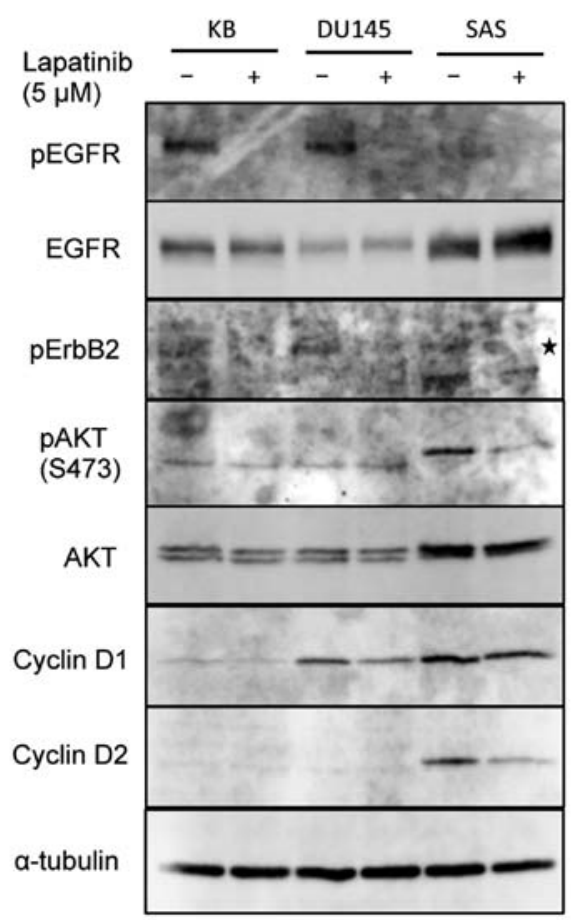

Figure 4. Lapatinib inhibits sphere formation of SAS cells via phosphorylation of AKT and cyclin D2 protein expression. (A) KB, DU145 and SAS cells $\left(1 \times 10^{3}\right)$ were cultured in lapatinib at the indicated concentrations on ultra-low attachment 6-well plates. (B) Cells were treated with lapatinib and the phosphorylation levels of EGFR, ErbB2 and AKT and protein expression level of cyclin D1 and D2 were determined by western blotting. Asterisk indicates the sieze of pErbB2 bands.

sphere formation (Fig. 3). The levels of EGFR phosphorylation in KB and SAS cells increased during sphere formation process after day 1 , but remained during sphere formation in DU145 cells. ErbB2 phosphorylation levels increased during sphere formation in all the cell lines. AKT phosphorylation increased during sphere formation of SAS cells but remained at low levels during sphere formation of KB and DU145 cells. Cyclin D1 levels were moderately altered during sphere formation in all cells. Cyclin D2 levels were increased along with sphere formation of SAS cells but were not detected in KB or DU145 spheres.

To determine whether sphere formation of lapatinib-resistant cell lines develops sensitivity to lapatinib, suspension cells were cultured in ultra-low attachment 6-well plates in medium with or without lapatinib. Sphere formation was inhibited by lapatinib in SAS cells, but not in KB or DU145 cells (Fig. 4A). Phosphorylation of EGFR and ErbB2 was reduced by lapatinib

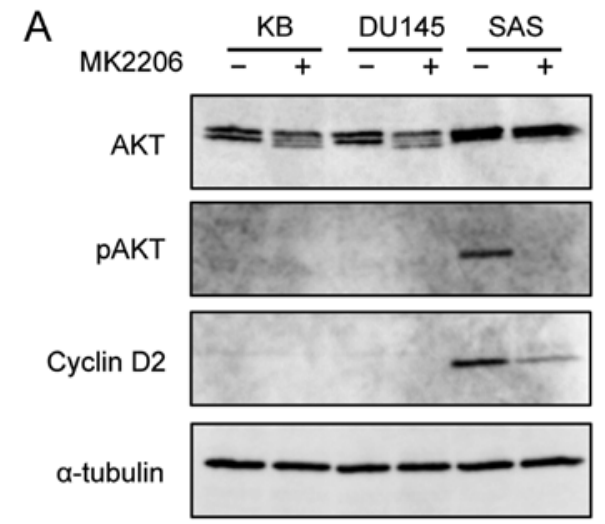

B
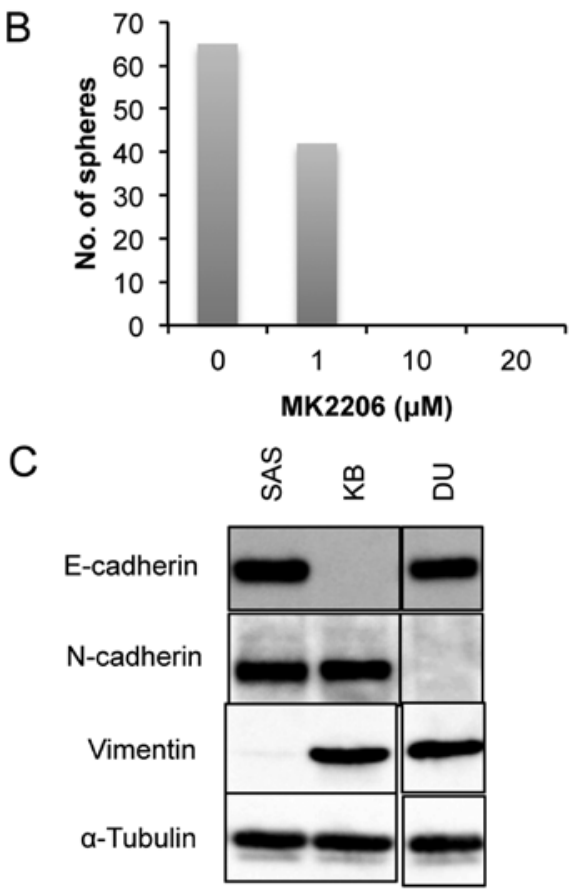

Figure 5. Sphere formation of SAS cells, showing epithelial properties, induced by AKT activation and cyclin D2 expression. (A) AKT phosphorylation and cyclin D2 expression levels in spheres from KB, DU145 and SAS cells in suspension cultures with or without MK2206 treatment were determined by western blotting. (B) Sphere cultures of SAS cells were treated with MK2206 for 6 days and the numbers of spheres was determined. (C) The epithelial marker E-cadherin or the mesenchymal markers $\mathrm{N}$-cadherin and vimentin were examined in SAS, KB and DU145 cells by western blotting.

in these cell lines (Fig. 4B). Furthermore, lapatinib treatment reduced AKT phosphorylation and cyclin D2 protein expression in SAS spheres. AKT phosphorylation and cyclin D2 protein expression were not detected in untreated KB and DU145 spheres. These results suggested that the ErbB/AKT/cyclin D2 pathway plays an important role in sphere formation of SAS cells.

To assess whether the AKT/cyclin D2 pathway is required for sphere formation of SAS cells, the effect of the AKT inhibitor MK2206 on cyclin D2 levels and SAS sphere formation was investigated. MK2206 inhibited AKT phosphorylation, leading to reduced protein levels of cyclin D2 (Fig. 5A). Furthermore, MK2206 suppressed sphere formation of SAS cells (Fig. 5B). Together, these results suggested that the ErbB/ $\mathrm{AKT} /$ cyclin D2 pathway plays a crucial role in sphere formation of SAS cells. 
EMT (mesenchymal-to-epithelial transition) is a characteristic of cancer stem cells or circulating tumour cells (24). To identify the differences in cell lines exhibiting sphere formation potency, we examined markers of epithelial and mesenchymal cells (Fig. 5C). KB cells were found to express the mesenchymal markers $\mathrm{N}$-cadherin and vimentin, but not the epithelial marker E-cadherin, indicating that KB cells are mesenchymal stem-like cells. DU145 cells expressed E-cadherin and vimentin, but not $\mathrm{N}$-cadherin, indicating that DU145 cells are meso-epithelial. SAS cells expressed E-cadherin, but not vimentin, indicating that SAS cells are epithelial cancer stem-like cells.

\section{Discussion}

Lapatinib, which can be orally administered, has been used to treat ErbB2-positive metastatic breast cancer patients as a dual TKI of both EGFR and ErbB2. In the present study, we found that lapatinib inhibited the proliferation of $\mathrm{HSC} 3, \mathrm{HSC} 4$ and Ca9-22 OSCC cell lines, in monolayer cultures. After lapatinib treatment, levels of AKT S473 phosphorylation and cyclin D1 protein expression, as well as phosphorylation of EGFR, ErbB2 and ErbB3, decreased in these cell lines, consistent with previous studies of breast cancer cells $(25,26)$. However, growth of OSCC cell lines KB and SAS, and the prostate cancer cell line DU145 were resistant to lapatinib in monolayer cultures. In breast cancer, the causes of lapatinib resistance involve both ErbB2-dependent and ErbB2-independent mechanisms. In the former case, expression and structural changes of ErbB2 induce lapatinib resistance $(27,28)$. In the latter case, activation of a pathway other than ErbB2 overcomes the inhibitory effects of lapatinib (19). In SAS cells, the phosphorylation levels of EGFR and ErbB2 were suppressed by lapatinib treatment, ErbB3 phosphorylation was not detected and AKT phosphorylation and cyclin D1 protein levels were not reduced by lapatinib treatment (Fig. 2). These results suggested that inhibition of the EGFR/ErbB2 pathway by lapatinib does not contribute to cell growth in SAS cells. However, EGFR, ErbB2, and ErbB3 were detected at low levels, and their phosphorylation levels were extremely low in KB and DU145 cells, indicating that their growth does not depend on ErbBs. Taken together, the results indicated that the lapatinib resistance of SAS, KB and DU145 cells is independent of ErbB2.

It has been reported that acquired resistance to lapatinib and AZD8931 result from EMT in breast cancer cell lines (19). These resistant cells express the mesenchymal markers vimentin and $\mathrm{N}$-cadherin, but not the epithelial marker E-cadherin. In the present study, lapatinib-resistant KB and DU145 cells were found to express vimentin, indicating that these cells possess mesenchymal properties. However, SAS cells were resistant to lapatinib, despite E-cadherin expression, and did not express vimentin. In addition, single cells from lapatinib-resistant cell lines KB, DU145 and SAS in ultra-low attachment wells survived, proliferated and formed aggregates, which were defined as spheres. Sphere forming potency is used as a surrogate in vitro method to isolate cancer stem cells (CSCs) from various human tumours (29-34). Thus, these cell lines potentially possess CSC properties. Together, these findings showed that resistance to lapatinib correlates with CSC potency, but not necessarily with the EMT.
Proliferation of SAS cells is resistant to lapatinib in anchorage-dependent cultures, but is sensitive in anchorage-independent cultures. In both culture conditions, EGFR and ErbB2 are expressed and phosphorylated, and phosphorylation levels are decreased by lapatinib. However, AKT phosphorylation is not affected by lapatinib treatment during anchorage-dependent growth but is reduced during anchorage-independent growth. These results suggested that different downstream effectors of the same receptor tyrosine kinases are involved in a culture condition-dependent manner. We previously reported that phosphorylation levels of EGFR and AKT were increased in SAS aggregate cultures, and were reduced by the lipid raft disruptor filipin III (20). Thus, it is possible that ErbB and PI3K/AKT colocalization to a specific region, such as membrane rafts, is induced by detachment of cells from substratum, resulting in ErbB/AKT signal transduction to promote sphere growth in SAS cells. In the present study, the phosphorylation levels of EGFR and ErbB2 were increased during sphere formation of lapatinib-resistant cell lines. In addition, AKT S473 phosphorylation and cyclin D2 protein levels were increased in SAS, but not in KB or DU145 cells. The AKT inhibitor MK2206 suppressed phosphorylation of AKT and cyclin D2 protein expression, thereby completely inhibiting sphere formation in SAS cells. These results indicated that the sensitivity of SAS spheres to lapatinib results from AKT phosphorylation and cyclin D2 expression.

Cancer cells possessing sphere formation ability survive and grow from single cells under anchorage-independent culture conditions. These characteristics are similar to the properties of circulating tumour cells (CTCs) during metastasis. CTCs are expected to be mesenchymal, since they enter the circulation from the primary tumour after EMT. However, CTCs consist of epithelial and mesenchymal subpopulations (35). Both CTC subpopulations colonize in distant organs, where they are involved in secondary tumourigenesis as epithelial CSCs (36). Thus, lapatinib can be used as a potential anticancer drug for both differentiated cancer cells of the primary tumour and for cells of the metastatic cancer.

\section{Acknowledgements}

The English in this manuscript has been edited by Textcheck. Funding for the present study was provided by Osaka University (M.N.) and Osaka Dental University (K.K.).

\section{References}

1. Olayioye MA, Neve RM, Lane HA and Hynes NE: The ErbB signaling network: Receptor heterodimerization in development and cancer. EMBO J 19: 3159-3167, 2000.

2. Schlessinger J: Cell signaling by receptor tyrosine kinases. Cell 103: 211-225, 2000.

3. Yarden Y and Sliwkowski MX: Untangling the ErbB signalling network. Nat Rev Mol Cell Biol 2: 127-137, 2001.

4. Herbst RS and Langer CJ: Epidermal growth factor receptors as a target for cancer treatment: The emerging role of IMC-C225 in the treatment of lung and head and neck cancers. Semin Oncol 29 (Suppl 4): S27-S36, 2002.

5. Kris MG, Natale RB, Herbst RS, Lynch TJ Jr, Prager D, Belani CP, Schiller JH, Kelly K, Spiridonidis H, Sandler A, et al: Efficacy of gefitinib, an inhibitor of the epidermal growth factor receptor tyrosine kinase, in symptomatic patients with non-small cell lung cancer: A randomized trial. JAMA 290: 2149-2158, 2003 
6. Cohen MH, Johnson JR, Chen YF, Sridhara R and Pazdur R: FDA drug approval summary: Erlotinib (Tarceva) tablets. Oncologist 10: 461-466, 2005.

7. Maheswaran S, Sequist LV, Nagrath S, Ulkus L, Brannigan B, Collura CV, Inserra E, Diederichs S, Iafrate AJ, Bell DW, et al: Detection of mutations in EGFR in circulating lung-cancer cells. N Engl J Med 359: 366-377, 2008.

8. Minguet J, Smith KH and Bramlage P: Targeted therapies for treatment of non-small cell lung cancer - Recent advances and future perspectives. Int J Cancer 138: 2549-2561, 2016.

9. Kokai Y, Myers JN, Wada T, Brown VI, LeVea CM, Davis JG, Dobashi K and Greene MI: Synergistic interaction of p185c-neu and the EGF receptor leads to transformation of rodent fibroblasts. Cell 58: 287-292, 1989.

10. Di Fiore PP, Pierce JH, Fleming TP, Hazan R, Ullrich A King CR, Schlessinger J and Aaronson SA: Overexpression of the human EGF receptor confers an EGF-dependent transformed phenotype to NIH 3T3 cells. Cell 51: 1063-1070, 1987.

11. Zhou Y, Li S, Hu YP, Wang J, Hauser J, Conway AN, Vinci MA Humphrey L, Zborowska E, Willson JK, et al: Blockade of EGFR and ErbB2 by the novel dual EGFR and ErbB2 tyrosine kinase inhibitor GW572016 sensitizes human colon carcinoma GEO cells to apoptosis. Cancer Res 66: 404-411, 2006.

12. Gomez HL, Doval DC, Chavez MA, Ang PC, Aziz Z, Nag S, $\mathrm{Ng} \mathrm{C}$, Franco SX, Chow LW, Arbushites MC, et al: Efficacy and safety of lapatinib as first-line therapy for ErbB2-amplified locally advanced or metastatic breast cancer. J Clin Oncol 26 2999-3005, 2008.

13. Esteva FJ, Yu D, Hung MC and Hortobagyi GN: Molecular predictors of response to trastuzumab and lapatinib in breast cancer. Nat Rev Clin Oncol 7: 98-107, 2010.

14. Liu L, Greger J, Shi H, Liu Y, Greshock J, Annan R, Halsey W, Sathe GM, Martin AM and Gilmer TM: Novel mechanism of lapatinib resistance in HER2-positive breast tumor cells: Activation of AXL. Cancer Res 69: 6871-6878, 2009.

15. Xia W, Bacus S, Husain I, Liu L, Zhao S, Liu Z, Moseley MA III, Thompson JW, Chen FL, Koch KM, et al: Resistance to ErbB2 tyrosine kinase inhibitors in breast cancer is mediated by calcium-dependent activation of RelA. Mol Cancer Ther 9: 292-299, 2010

16. Zoppoli G, Moran E, Soncini D, Cea M, Garuti A, Rocco I, Cirmena G, Grillo V, Bagnasco L, Icardi G, et al: Ras-induced resistance to lapatinib is overcome by MEK inhibition. Curr Cancer Drug Targets 10: 168-175, 2010.

17. Martin AP, Mitchell C, Rahmani M, Nephew KP, Grant S and Dent P: Inhibition of MCL-1 enhances lapatinib toxicity and overcomes lapatinib resistance via BAK-dependent autophagy. Cancer Biol Ther 8: 2084-2096, 2009.

18. Martin AP, Miller A, Emad L, Rahmani M, Walker T, Mitchell C, Hagan MP, Park MA, Yacoub A, Fisher PB, et al: Lapatinib resistance in HCT116 cells is mediated by elevated MCL-1 expression and decreased BAK activation and not by ERBB receptor kinase mutation. Mol Pharmacol 74: 807-822, 2008.

19. Creedon H, Gómez-Cuadrado L, Tarnauskaitė Ž, Balla J, Canel M, MacLeod KG, Serrels B, Fraser C, Unciti-Broceta A, Tracey $\mathrm{N}$, et al: Identification of novel pathways linking epithelial-to-mesenchymal transition with resistance to HER2-targeted therapy. Oncotarget 7: 11539-11552, 2016

20. Ohnishi Y, Yasui H,KakudoKand Nozaki M:Cetuximab-resistant oral squamous cell carcinoma cells become sensitive in anchorage-independent culture conditions through the activation of the EGFR/AKT pathway. Int J Oncol 47: 2165-2172, 2015.

21. Farnie G, Johnson RL, Williams KE, Clarke RB and Bundred NJ: Lapatinib inhibits stem/progenitor proliferation in preclinical in vitro models of ductal carcinoma in situ (DCIS). Cell Cycle 13: $418-425,2014$
22. Caldon CE, Daly RJ, Sutherland RL and Musgrove EA: Cell cycle control in breast cancer cells. J Cell Biochem 97: 261-274, 2006.

23. Lange CA and Yee D: Killing the second messenger: Targeting loss of cell cycle control in endocrine-resistant breast cancer. Endocr Relat Cancer 18: C19-C24, 2011.

24. Tsai JH and Yang J: Epithelial-mesenchymal plasticity in carcinoma metastasis. Genes Dev 27: 2192-2206, 2013.

25. Rusnak DW, Lackey K, Affleck K, Wood ER, Alligood KJ, Rhodes N, Keith BR, Murray DM, Knight WB, Mullin RJ, et al: The effects of the novel, reversible epidermal growth factor receptor/ErbB-2 tyrosine kinase inhibitor, GW2016, on the growth of human normal and tumor-derived cell lines in vitro and in vivo. Mol Cancer Ther 1: 85-94, 2001.

26. Spector NL, Xia W, Burris H III, Hurwitz H, Dees EC, Dowlati A, O'Neil B, Overmoyer B, Marcom PK, Blackwell KL, et al: Study of the biologic effects of lapatinib, a reversible inhibitor of ErbB1 and ErbB2 tyrosine kinases, on tumor growth and survival pathways in patients with advanced malignancies. J Clin Oncol 23: 2502-2512, 2005.

27. Trowe T, Boukouvala S, Calkins K, Cutler RE Jr, Fong R, Funke R, Gendreau SB, Kim YD, Miller N, Woolfrey JR, et al: EXEL-7647 inhibits mutant forms of ErbB2 associated with lapatinib resistance and neoplastic transformation. Clin Cancer Res 14: 2465-2475, 2008

28. Kancha RK, von Bubnoff N, Bartosch N, Peschel C, Engh RA and Duyster J: Differential sensitivity of ERBB2 kinase domain mutations towards lapatinib. PLoS One 6: e26760, 2011.

29. Dontu G, Abdallah WM, Foley JM, Jackson KW, Clarke MF, Kawamura MJ and Wicha MS: In vitro propagation and transcriptional profiling of human mammary stem/progenitor cells. Genes Dev 17: 1253-1270, 2003.

30. Singh SK, Hawkins C, Clarke ID, Squire JA, Bayani J, Hide T, Henkelman RM, Cusimano MD and Dirks PB: Identification of human brain tumour initiating cells. Nature 432: 396-401, 2004

31. Ponti D, Costa A, Zaffaroni N, Pratesi G, Petrangolini G, Coradini D, Pilotti S, Pierotti MA and Daidone MG: Isolation and in vitro propagation of tumorigenic breast cancer cells with stem/progenitor cell properties. Cancer Res 65: 5506-5511, 2005.

32. Ricci-Vitiani L, Lombardi DG, Pilozzi E, Biffoni M, Todaro M, Peschle C and De Maria R: Identification and expansion of human colon-cancer-initiating cells. Nature 445: 111-115, 2007.

33. Eramo A, Lotti F, Sette G, Pilozzi E, Biffoni M, Di Virgilio A, Conticello C, Ruco L, Peschle C and De Maria R: Identification and expansion of the tumorigenic lung cancer stem cell population. Cell Death Differ 15: 504-514, 2008.

34. Han ME, Jeon TY, Hwang SH, Lee YS, Kim HJ, Shim HE, Yoon S, Baek SY, Kim BS, Kang CD, et al: Cancer spheres from gastric cancer patients provide an ideal model system for cancer stem cell research. Cell Mol Life Sci 68: 3589-3605, 2011.

35. Yu M, Bardia A, Wittner BS, Stott SL, Smas ME, Ting DT, Isakoff SJ, Ciciliano JC, Wells MN, Shah AM, et al: Circulating breast tumor cells exhibit dynamic changes in epithelial and mesenchymal composition. Science 339: 580-584, 2013.

36. Beerling E, Seinstra D, de Wit E, Kester L, van der Velden D, Maynard C, Schäfer R, van Diest P, Voest E, van Oudenaarden A, et al: Plasticity between epithelial and mesenchymal states unlinks EMT from metastasis-enhancing stem cell capacity. Cell Rep 14: 2281-2288, 2016. 